\title{
DISTRIBUTED WAVELENGTH RESERVATION METHOD FOR FAST LIGHTPATH SETUP IN WDM NETWORKS
}

\author{
Yosuke Kanitani, ${ }^{1}$ Shin' ichi Arakawa, ${ }^{2}$ Masayuki Murata, ${ }^{3}$ and Ken-ichi Kitayama ${ }^{1}$ \\ ${ }^{1}$ Graduate School of Engineering, Osaka University, \\ 2-1 Yamadaoka, Suita, Osaka 565-0871, Japan \\ ${ }^{2}$ Graduate School of Economics, Osaka University, \\ 1-7 Machikaneyama, Toyonaka, Osaka, 560-0043, Japan \\ ${ }^{3}$ Graduate School of Information Science and Technology, Osaka University, \\ 1-5 Yamadaoka, Suita, Osaka 565-0871, Japan
}

\begin{abstract}
A promising approach to the effective utilization of wavelength division multiplexed networks is to transfer data on an on-demand basis using fast wavelength reservation. Data can then be transferred using the assigned wavelength channel. However, if wavelength reservation fails, the lightpath setup delay, which is defined as the time from when the data-transfer request arises at the source node to when the lightpath between the source-destination pair is successfully established, is seriously affected since retrials of wavelength reservation are in turn delayed by propagation delays. In this paper, we propose a new wavelength reservation method to reduce lightpath setup delay. Whereas conventional methods reserve a wavelength in either the forward or backward direction, we propose to reserve it in both directions. We used computer simulations to compare our proposed method with existing methods. The results showed that our method was more efficient except under high traffic loads.
\end{abstract}

\section{INTRODUCTION}

A promising approach to the effective utilization of wavelength division multiplexed (WDM) networks is to transfer the data on an on-demand basis. That is, when a data request arises at a source node, a wavelength is dynamically reserved between the source and destination nodes, and a wavelength channel (called a lightpath [1]) is configured. After the data is transferred using the lightpath, the wavelength is immediately released. 
Two methods have previously been presented to set up lightpaths in a distributed manner [2]. In both methods, the lightpaths are established by exchanging control packets between the source and destination nodes. The actual reservation of the link resources is performed while the control packet is traveling from either the source node to the destination node (i.e., forward direction), or from the destination node to the source node (i.e., backward direction). There have been several studies on reservation schemes aimed at reducing the blocking probability for lightpath requests [2-6]. However, a more important measure for these reservation models is lightpath setup delay, which is defined as the time from when the lightpath request arrives at the source node to when a lightpath is successfully configured between the source and destination nodes. Only in [6], lightpath setup delay with the retrial is evaluated but this retrial just employs existing method repeatedly and an improvement method of lightpath setup delay including the retrial is not considered. However, in order to transfer the data, the source node must keep trying to setup a lightpath until the lightpath is successfully configured. Consequently, lightpath setup delay is increased by such retrials due to the link propagation delay along the path. Thus, it is important to improve lightpath setup delay with consideration of retrials.

In this paper, we propose a new wavelength reservation method aimed at reducing lightpath setup delay by increasing the trials of wavelength reservation. More specifically, by integrating two existing reservation method, our method reserve a wavelength in both forward and backward direction, while existing reservation methods reserve a wavelength in either forward or backward direction.

The rest of the paper is organized as follows. Section 2 outlines wavelengthrouted networks and related work, Section 3 presents our proposed method, Section 4 presents the simulation results, and Section 5 includes a brief summary.

\section{RELATED WORKS}

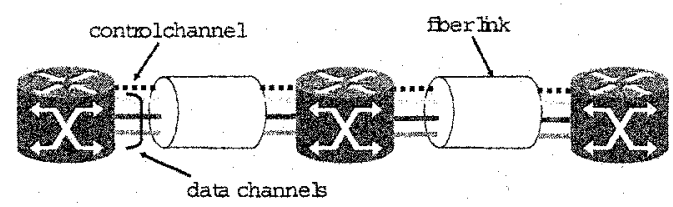

Figure 1 . Wavelength routed network 
First, we will describe the structure of our wavelength-routed network. A model of the network is shown in Figure 1. It consists of optical crossconnects (OXCs) and optical fibers. Each fiber carries a certain set of wavelengths. Within these sets, one wavelength carries control packets and the other wavelengths are used for data transfer. The control packet controls the setup and/or tear down of lightpaths. Conventional lightpath setup methods for wavelength-routed networks are mainly based on two reservation schemes: forward reservation and backward reservation. In forward reservation, the source node sends a reservation packet (RESV) immediately when a lightpath request arises. The reservation packet reserves a wavelength from the source node to the destination node. Since the source node has no information on wavelength availability, there is no guarantee that a wavelength will be available in each link along the path. In backward reservation, the source node sends a probe packet (PROBE) toward the destination node. Information on usage of wavelengths along the forward path is collected, but no wavelengths are reserved at this time. Each intermediate node on the forward path only removes wavelengths from the list if those wavelengths are currently in use. Based on the information from the probe packet, the destination node determines a wavelength for reservation, and then sends a RESV packet toward the source node. These forward and backward reservation schemes are illustrated in Figures 2 and 3. Note that, in this paper, we do not consider wavelength conversion facilities. That is, a lightpath uses the same wavelength along the entire path, which is known as the wavelength continuity constraint [7].

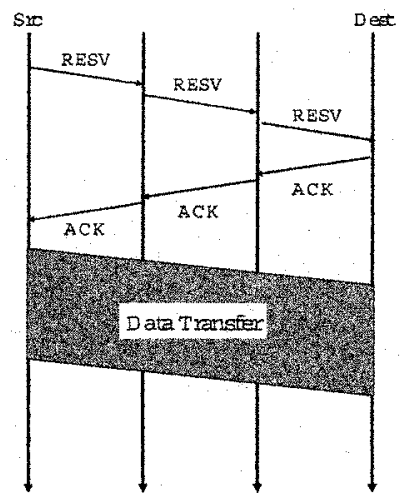

Figure 2. Forward Reservation

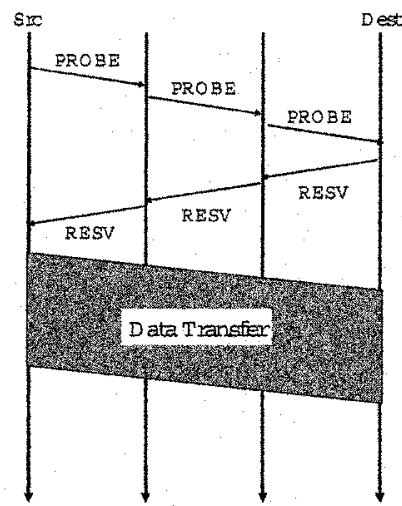

Figure 3. Backward Reservation 


\section{OUR PROPOSAL}

In both these existing reservation methods, there is only one trial for lightpath establishment during the round-trip propagation time. We therefore propose a new method for lightpath setup, based on integrating the forward and backward reservation schemes, which tries to establish a lightpath twice during the round-trip propagation time. Figures 4 and 5 illustrate our proposed scheme. In this scheme, when a lightpath setup request arises at a source node, the source node sends a PROBE packet toward the destination node, as in backward reservation. However, in contrast to backward reservation, when the destination node receives a PROBE packet, it sends not only a RESV packet (or NACK packet) but also a PROBE packet toward the source node. The PROBE packet collects information on wavelength usage from the destination node to the source node, and the source node selects a wavelength based on this information. This retrial scenario is illustrated in Figure 5. The main feature of the proposed scheme is that the edge nodes exchange PROBE packets. Below we explain the details of our proposed reservation scheme.

1. Behavior of the source node

(S1) When a data transfer request arrives from a terminal, the source node creates a PROBE packet and sends it toward the destination node.

(S2) When a RESV (or ACK) packet arrives, the source node informs the terminal that a lightpath has been established.

(S3) When a NACK packet arrives, the source node sends a RESV packet and a PROBE packet. (A NACK packet is always accompanied by a PROBE packet.) This is what happens in the case of reservation failure in the backward direction; the original features of our proposal relate to this behavior. In addition, if a reservation is blocked halfway, the source node must also send a release packet (RLS).

(S4) When the data transfer is completed, the source node sends a RLS packet to tear down the lightpath.

2. Behavior of intermediate node(s)

(I1) When an intermediate node receives a PROBE packet, it calculates the intersection between a probed wavelength group and a wavelength group that is available in the next link.

(I2) When a RESV or RLS packet arrives, an intermediate node reserves or releases the wavelength, respectively.

(I3) An ACK and a NACK packet are forwarded to the next node without any processing. 
3. Behavior of the destination node

(D1) Basically, the behavior of the destination node is similar to that of the source node. When a PROBE packet arrives, the destination node sends RESV and PROBE packets simultaneously.

(D2) When a NACK packet arrives, the destination node sends RESV, PROBE, and RLS packets simultaneously. This is similar to (S3).

(D3) When a RSV packet arrives, the destination node sends an ACK packet toward the source node to notify that a lightpath has been established.
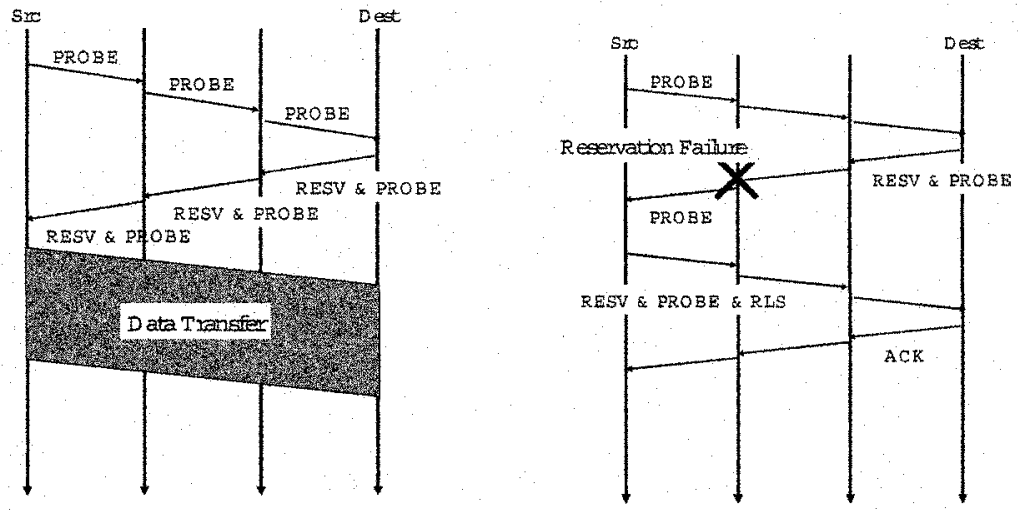

Figure 4. Proposed scheme (successful case) Figure 5. Proposed scheme (retrial case)

\section{SIMULATION RESULTS}

\subsection{Simulation model}

To evaluate the performance of our proposed scheme, we used computer simulation to compare it with a backward reservation scheme. We used a random network as the simulation topology. Figure 6 shows the topology, which has 15 nodes. Other simulation parameters are briefly described below.

- The number of wavelengths on each link is set to 32.

- Each link has a random propagation delay with mean $1.77[\mathrm{~ms}]$.

- Data-transfer requests arrive according to a Poisson process, and the lightpath is held for a connection-holding period that is assumed to be exponentially distributed with mean $1 / \mu[m s]$. 


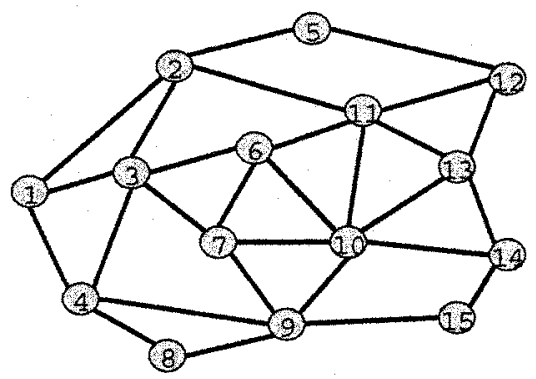

Figure 6. Random Network

We define the load $\rho_{n p}$ in Figures 7 and 8 as the offered load for a sourcedestination node pair.

\subsection{Evaluation of Lightpath Setup Delay}
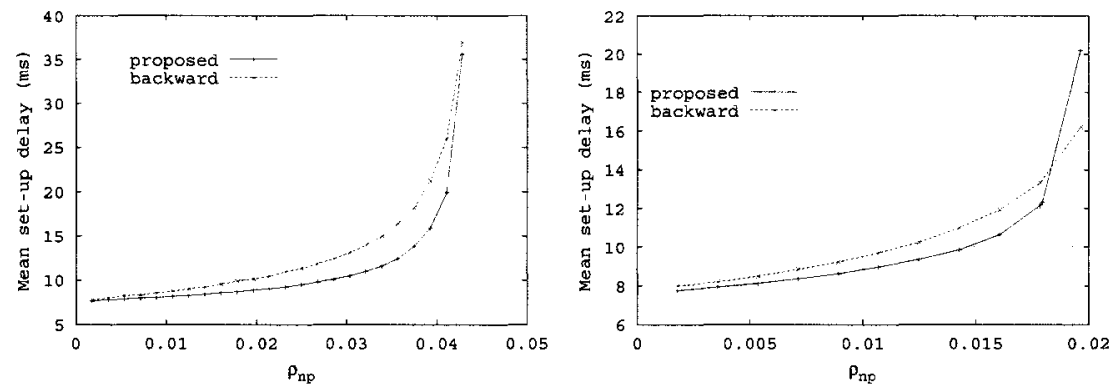

Figure 7. Lightpath setup delay $(1 / \mu=$ Figure 8 . Lightpath setup delay $(1 / \mu=$ $100 \mathrm{~ms})$ $10 \mathrm{~ms}$ )

In Figures 7 and Figure 8, we present the mean setup delay dependent on the load $\rho_{n p}$. Figure 7 and Figure 8 show the results when the average of holding time $1 / \mu$ is set to $100[\mathrm{~ms}]$ and $10[\mathrm{~ms}]$, respectively. The results showed that our proposed scheme performed better than backward reservation at almost every range, except for $\rho_{n p}>0.018$ in Figure 8. The proposed scheme was inferior to backward reservation in this situation because of the inaccuracy of the probed information. When the information collected by a PROBE packet is not accurate due to link propagation delay, the information becomes outof-date. If the information is too old, the edge node may select a wavelength that has already been reserved by another node-pair. In the proposed scheme, 
the edge nodes can make several attempts to send PROBE packets compared with backward reservation, so the accuracy of the information is more important. When the connection-holding period $1 / \mu$ is relatively short compared to the link propagation delay (as in Figure 8), the information tends to be less accurate.

\subsection{Variation in Lightpath Setup Delay}

In a wavelength-routed network, it is preferable to establish a lightpath with little variation in setup delay. If a wavelength reservation method has large variation, it is difficult to achieve stable data transmission. Therefore, it is important to consider variations in lightpath setup delay. In this section, we use the standard deviation (STD) as an indication of variation in delay.

We describe the statistical properties of $1 / \mu=100[\mathrm{~ms}]$ as an example. These include mean setup delay (Mean) and STD. Table 1 and Table 2 summarize these characteristics dependent on the number of hop-counts " $\mathrm{H}$ " of each source-destination node pair.

These tables indicate that the STD resulting from our proposed method is about half that resulting from backward reservation because our method can setup a lightpath in both forward and backward directions. In relation to the discussion in section 4.2 , our scheme performs better, especially under a low traffic load.

Table 1. Variations in lightpath setup delay in proposed scheme

\begin{tabular}{|c||c|c||c|c||c|c||c|c|}
\hline \multicolumn{1}{|c||}{} & \multicolumn{2}{c||}{$\mathrm{H}=1$} & \multicolumn{2}{c||}{$\mathrm{H}=2$} & \multicolumn{2}{c||}{$\mathrm{H}=3$} & \multicolumn{2}{c|}{$\mathrm{H}=4$} \\
\hline$\rho_{n p}$ & Mean & STD & Mean & STD & Mean & STD & Mean & STD \\
\hline 0.0018 & 3.60 & 3.72 & 10.43 & 4.11 & 20.35 & 11.45 & 43.70 & 14.66 \\
0.0125 & 3.82 & 6.57 & 11.17 & 8.02 & 21.91 & 11.22 & 47.31 & 14.93 \\
0.0232 & 4.11 & 9.57 & 12.12 & 10.80 & 24.30 & 15.59 & 53.48 & 21.82 \\
0.0339 & 4.62 & 11.99 & 13.89 & 14.19 & 29.65 & 22.71 & 68.79 & 36.07 \\
\hline
\end{tabular}

Table 2. Variations in lightpath setup delay in backward reservation

\begin{tabular}{|c||c|c||c|c||c|c||c|c|}
\hline \multicolumn{1}{|c||}{} & \multicolumn{2}{c||}{$\mathrm{H}=1$} & \multicolumn{2}{c||}{$\mathrm{H}=2$} & \multicolumn{2}{c||}{$\mathrm{H}=3$} & \multicolumn{2}{c|}{$\mathrm{H}=4$} \\
\hline$\rho_{n p}$ & Mean & STD & Mean & STD & Mean & STD & Mean & STD \\
\hline 0.0018 & 3.57 & 7.02 & 10.53 & 17.94 & 20.79 & 25.86 & 45.52 & 27.39 \\
0.0125 & 4.08 & 13.18 & 11.92 & 15.39 & 23.73 & 22.33 & 51.59 & 27.83 \\
0.0232 & 4.64 & 19.74 & 13.79 & 21.72 & 28.26 & 32.36 & 63.19 & 41.91 \\
0.0339 & 6.30 & 23.16 & 17.02 & 28.88 & 37.91 & 44.92 & 89.51 & 65.99 \\
\hline
\end{tabular}




\section{SUMMARY}

In this paper, we presented a new lightpath setup method that reserves wavelengths in both forward and backward directions. The main objective of our method is to reduce lightpath setup delay. Our proposed method, which integrates features of two existing methods, performs lightpath establishment twice within a round-trip, while the previous methods perform it only once. The simulation results indicate that the proposed method performs better except under high traffic loads. We also evaluated other statistical properties of the methods. The results showed that the standard deviation of our method was much smaller than that of the conventional method. In future work, we plan to develop a numerical analysis of lightpath setup delay.

\section{REFERENCES}

[1] I. Chlamtac, A. Ganz, and G. Karmi, "Lightpath communications: An approach to high bandwidth optical WAN's," IEEE Transactions on Communications., vol. 40, pp. 11711182, July 1992.

[2] X. Yuan, R. Gupta, R. Melhem, R. Gupta, Y.Mei, and C. Qiao, "Distributed control protocols for wavelength reservation and their performance evaluation," Photonic Network Communications, vol. 1, pp. 207-218, November 1999.

[3] L. Pezoulas, M. J. Francisco, I. Lambadaris, and C. Huang, "Performance analysis of a backward reservation protocol in networks with sparse wavelength conversion," in Proceedings of IEEE International Conference on Communications, vol. 2, pp. 1468-1473, May 2003.

[4] F. Feng, X. Zheng, H. Zhang, and Y. Guo, "An efficient distributed control scheme for lightpath establishment in dynamic wdm networks," Photonic Network Communications, vol. 7, pp. 5-15, January 2004.

[5] A. V. Shichani and H. T. Mouftah, "A novel distributed progressive reservation protocol for WDM all-optical networks," in Proceedings of IEEE International Conference on Communications, vol. 2, pp. 1463-1467, May 2003.

[6] D. Saha, "An efficient wavelength reservation protocol for establishment in all-optical networks (AONs)," in Proceedings of IEEE Global Telecommunications Conference, vol. 2, pp. 1264-1268, November 2000.

[7] Z. Zhang, J. Fu, and D. G. L. Zhang, "Lightpath routing for intelligent optical networks," IEEE Network, vol. 15, pp. 28-35, July 2001. 